\title{
Uterine Leiomyosarcoma
}

\author{
Ingolf Juhasz-Böss ${ }^{a} \quad$ Lena Gabriela $^{a}$ Rainer M. Bohle ${ }^{b}$ Lars C. Horn ${ }^{c}$ \\ Erich-Franz Solomayer ${ }^{a}$ Georg-Peter Breitbach ${ }^{a}$
}

\author{
${ }^{a}$ Department of Gynecology, Obstetrics and Reproductive Medicine, Saarland University, Homburg/Saar, Germany; \\ ${ }^{b}$ Department of Pathology, Saarland University, Homburg/Saar, Germany; \\ ${ }^{\mathrm{c}}$ Division of Gynecologic Pathology, Department of Pathology, Leipzig University, Leipzig, Germany
}

\author{
Keywords \\ Leiomyosarcoma · Uterus · Diagnosis · Surgery · \\ Therapy
}

\section{Summary}

Uterine leiomyosarcoma (uLMS) is a rare entity among malignant gynecologic tumors with a very unfavorable prognosis and the highest prevalence in the pre- and peri-menopause. Only early-stage tumors have an acceptable prognosis, provided the patient has been treated without injuring the uterus. uLMS is often diagnosed accidentally and the correct diagnosis ishampered by equivocal features similar to the far more frequent benign uterine fibroids. Surgery is the basis of therapy, and it should be done in order to remove the uterus intact. As vaginal, abdominal, and endoscopic surgery - possibly including morcellation - are the methods of choice for the treatment of uterine fibroids, pre-operatively undiagnosed leiomyosarcoma detected by pathologic examination will have a worsened prognosis. Systemic treatment and radiotherapy are of no proven value in the adjuvant setting. Thus, there is strong need for a reliable pre-operative risk score for leiomyosarcoma in order to justify diagnostic means beyond clinical routine and to choose the correct surgical pathway. The clinical problems in the diagnosis of leiomyosarcoma and treatment are exemplified by a case report of a 30-year-old childless patient. Diagnostic tools as well as treatment options in adjuvant and palliative situations are reviewed.

(C) 2018 S. Karger GmbH, Freiburg

\section{Introduction}

With of $60-70 \%$ uterine leiomyosarcoma (uLMS) represents the main entity of the heterogeneous group of uterine sarcomas (US)
[1]. Admittedly it accounts for only $1 \%$ of all uterine malignancies, but is most dangerous as it implies a very poor prognosis. It may be considered as a separate entity for a number of reasons, e.g. particular histopathologic features and the possible diagnostic confusion with uterine leiomyoma (uLM) and STUMP (smooth muscle tumors of uncertain malignant potential). uLMS occurs at a median age of 50 years and most patients are perimenopausal [2]. Typically large pelvic masses are found, which may cause hemorrhage or symptoms of vaginal or abdominal pressure. Nevertheless, many patients remain asymptomatic. uLMS is rarely suspected before operation and more often detected accidentally after removing the uterus or single uLM with or without morcellation. Although absolute numbers of uLMS detected post-surgically are small - numbers in the literature range from 1:498 [3] to 1:1,851 [4] out of all uterine myoma interventions - inadequate operation is considered detrimental for the individual patient and should be prevented.

\section{Case Report}

Characteristic diagnostic and therapeutic problems of uLMS are elucidated by the case of a 30-year-old patient (nulligravida) who was referred to our hospital due to a rapidly growing uterus without abnormal bleeding under oral contraconception in a long-cycle schedule. Except for an enlarged, but still mobile uterus gynecological examination was normal. Transvaginal sonography (TVS) showed a paracervical tumor of $5.7 \times 5.4 \times 3.8 \mathrm{~cm}$ in diameter at the dorsal uterine wall without signs of intratumoral necrosis (fig. 1a). To exclude the differential diagnosis of uLMS, an additional MRI (magnetic resonance imaging) of the pelvis was performed which seemed to clear the suspicion of uLMS. Due to her prospective desire to have children, the patient shared the therapeutic decision of endoscopical enucleation of the tumor. Intraoperatively, the uterus presented with a widespread distension of the posterior wall (fig. 1b); the tumor mass was enucleated and morcellated, the uterine wall being carefully reconstructed afterwards. No peri- or post-operative complications were reported.

Histopathologic examination revealed muscle cell proliferations with highgrade nuclear atypia and a mitotic index of 7 out of 10 / high power field (HPF), but no description of tumor cell necrosis. This resulted in the diagnosis STUMP (fig. 1c+d). Pathologic reference re-evaluation described lacking evidence of uLMS and favored STUMP as well. The patient was dismissed after thorough

\section{KARGER}

() 2018 S. Karger GmbH, Freiburg

Fax +497614520714
Prof. Dr. med. Ingolf Juhasz-Böss Department of Gynecology and Obstetrics Saarland University

Kirrbergerstraße 100, 66424 Homburg/Saar, Germany ingolf.juhasz-boess@uks.eu 

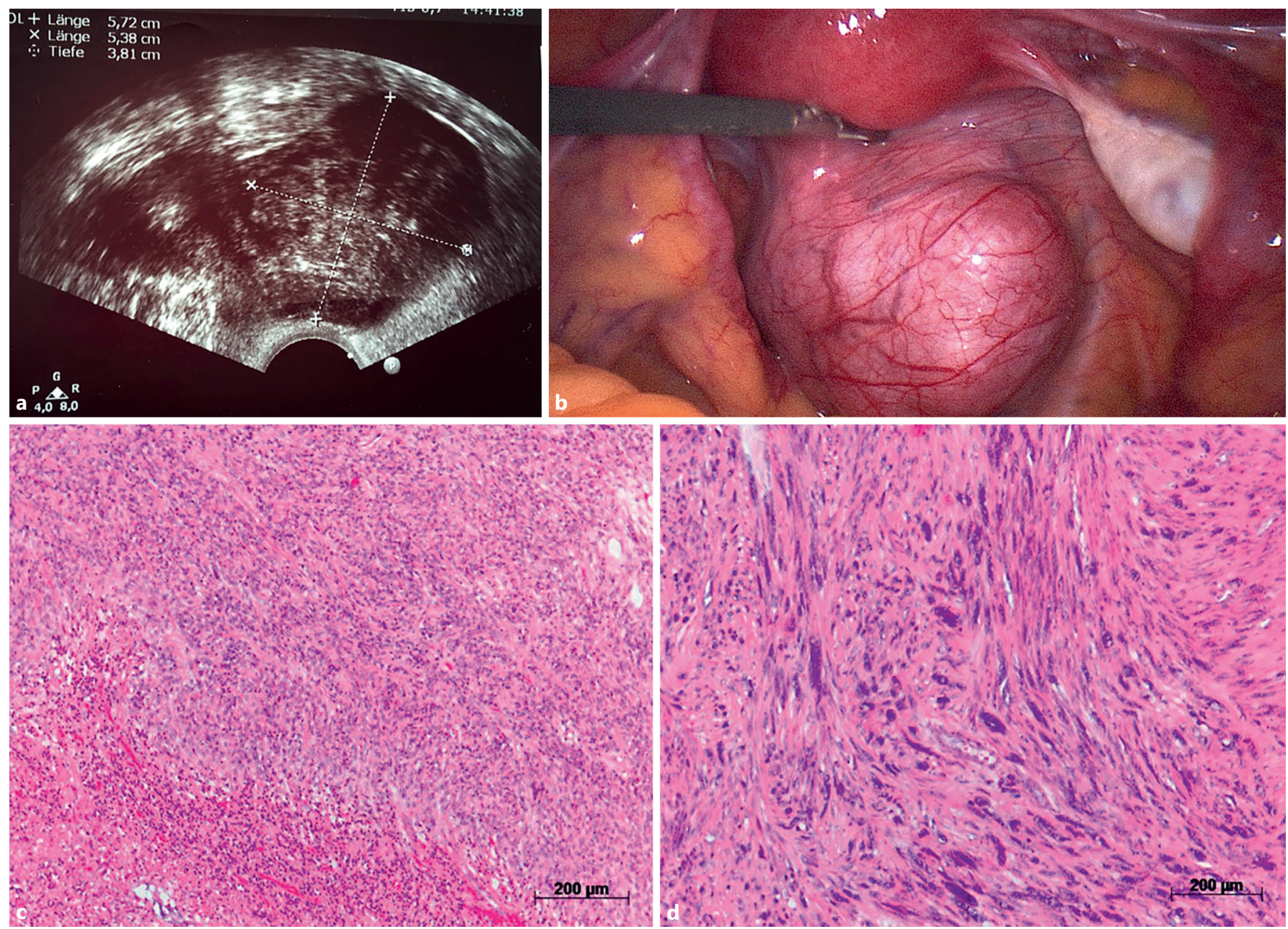

Fig. 1. Presented case report at diagnosis of STUMP. a Sonographic view of the uterine tumor. b Intraoperative view of the retrouterine tumor. c Histopathologic examination revealed muscle cell proliferations with focal tumor cell necrosis (100×, HE). d High-grade nuclear atypias (100×, HE).

discussion of the diagnosis; advice was given to consider finishing contraception and aiming at a near-term pregnancy. 3-monthly intervals were proposed for follow-up examination.

After 11 months the patient - still under contraception - was presented again with suspicion of local relapse. Gynecological examination, TVS and MRI (fig. 2a) showed irregular tumor masses at the dorsal uterine wall. Subsequently shared decision was made to remove the uterus completely despite the existing desire to have children. During abdominal surgery metastases in the pelvic peritoneum and in the abdominal wall (trokar sites) were found which were completely resected. Histopathologic examination now definitely showed uLMS with spindle cells, high mitotic activity $>15 / 10 \mathrm{HPF}$ and large areas of tumor cell necrosis (fig. $2 \mathrm{~b}+\mathrm{c}$ ). Again there were no peri- or post-operative complications. The patient had 5 cycles (refused 1) of post-operative doxorubicin / ifosfamide chemotherapy $\mathrm{q} 3 \mathrm{w}$, a negative second-look surgery, and a consolidation radiotherapy of the pelvis. After a follow-up of 22 months the patient is still without recurrence.

The reported disease process raises some issues of general interest, which will be discussed later on.

\section{Diagnosis}

As uLMS mimics benign leiomyoma (uLM) its diagnosis is a crucial challenge. Common opinion is that neither imaging proce- dures, such as TVS, CT-Scan and MRI, nor a pre-operative dilation and curettage (D\&C), nor endometrial biopsy (EMB) will contribute to better discrimination of benign and malignant tumors. Nevertheless, there is some evidence [5] that D\&C as well as EMB may detect invasive cancer to a considerable percentage ( $86 \%$ for either method), which does not differ from the detection rate of endometrial cancer.

Furthermore there are hints from small study populations that more sophisticated techniques, such as contrast-enhanced and balance-weighed dynamic MRI $[6,7,8]$ may distinguish uLM, STUMP, and uLMS. However, it seems difficult to use those particular methods in a clinical routine setting. This also applies to PET-CT, even if in 5 out of 5 patients the correct diagnosis was predicted pre-operatively [9].

Thus, the challenge remains to find epidemiological, clinical, and sonographic parameters to establish a helpful risk score for the selection of patients who need more subtle pre-operative imaging techniques and D\&C. Köhler et al. [4, 10] have analyzed anamnestic and clinical criteria of 227 uLMS and 3920 uLM and found (among others) the following discriminating items:

- postmenopausal status and/or postmenopausal bleeding, 

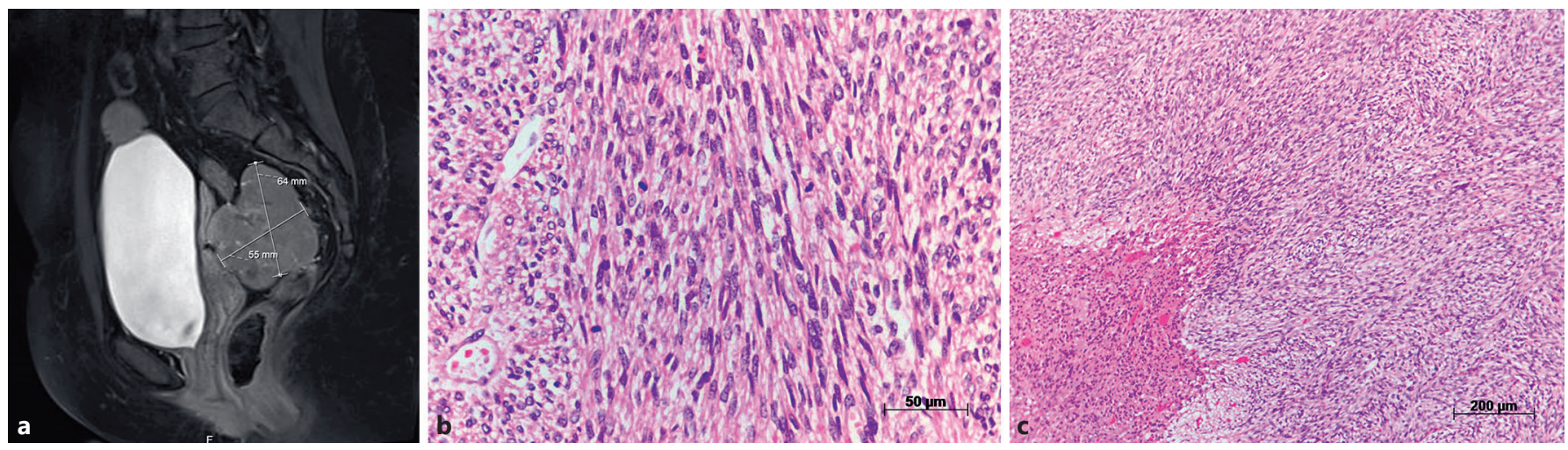

Fig. 2. Presented case report with recurrent pelvic tumor and diagnosis of uterine leimyosarcoma (uLMS). a MRI with retrouterine pelvic mass (uLMS), 11 months after removal of STUMP. b,c Histopathologic examination now definitely showed uLMS with spindle cells, high mitotic activity $>15 / 10 \mathrm{HPF}$ and large areas of tumor cell necrosis (b 100×, c 400x, HE).

- abnormal premenopausal bleeding,

- suspicious sonographic findings,

- rapid tumor growth and age $>45$ years,

- tumor size $>8 \mathrm{~cm}$.

A risk score for uLMS has been developed [4] and is currently under evaluation. With the help of a conclusively evaluated risk score the number of patients who need extensive (and expensive) non-routine pre-operative diagnosis might be substantially restricted.

\section{Surgical Therapy}

Complete removal of the uterus is obligatory for the surgical management of uLMS. Bilateral salpingo-oophorectomy (BSO) may be considered, depending on menopausal status. Ovaries may be preserved in young patients with tumors confined to the uterus $[2,11,12]$. Intraperitoneal morcellation of uLMS is associated with poorer prognosis; endoscopic supracervical hysterectomy or tumor enucleation and morcellation should be avoided $[13,14]$. The incidence of pelvic and para-aortic lymph-node metastasis is low; if metastases are palpable, hematogeneous metastasis is likely so that widespread that lymphonodeectomy will not improve the prognosis and is not advisable [15]. Fertility preservation should not be recommended, as only limited data is available.

Diagnosis of uLMS after partial uterine resection should prompt instantaneous completion surgery. After a median interval of 6 weeks, $64 \%$ persisting uLMS and 38\% STUMP are found [16]. In advanced situations, a debulking operation should be attempted [11].

\section{Prognosis}

uLMS is a highly aggressive tumor and implies dismal prognosis independent of grading, even if the tumor is confined to the uterus. Consequently, the World Health Organization (WHO) and Gynecologic Oncology Group (GOG) do not recommend grading of
uLMS anymore. Relapse rates are reported in between 53 and 71\% after 5 years. Norwegian data show a 5 -year overall survival (OAS) no better than $51 \%$ even in stage I tumors and $25 \%$ in stage II [17]. Only early-stage tumors (IA) seem have a more favorable prognosis, with an OAS of $76.6 \%$ [12], whereas stage IB tumors are significantly worse with an OAS of $48.4 \%$. Prognostic factors are age, tumor stage, and tumor size. OAS will decrease depending on tumor size: $<5 \mathrm{~cm}$ (76.6\%), 5-10 cm (52.9\%) and > $10 \mathrm{~cm}(41.9 \%)$. Involvement of the uterine cervix will reduce OAS to 28.5 vs. $55.3 \%$ without cervical involvement [12]. Additional prognostic factors under discussion are free margins, mitotic score, and vessel invasion [18]. Morcellation truly contributes to grave prognosis [14]. As pulmonal metastasis is common, a thoracic X-ray image or CTscan should be considered.

\section{Histopathologic Diagnosis and Staging}

Most uLMS are found within the uterine wall as single tumors; in case of multiple uterine leimyomas the largest tumor should be examined carefully to exclude malignant degeneration. The tumor surface often appears grayish yellow or red and has a soft or fleshy consistency. Hemorrhagic and necrotic areas may be seen macroscopically. Typically uLMS shows characteristic hypercellular spindle cells, diffuse moderate to serious cell atypia, a high mitotic index $>$ 15/10 HPF, atypical mitosis and tumor cell necrosis [19]. Determination of necrotic type gains importance; coagulative tumor cell necrosis should be distinguished from infarction related to hyalinic necrosis. Tumor cell necrosis is the main criterion in malign leiomyogenic tumors, followed by infiltrating growth into the myometrium with blurry margins, extrauterine spread and tumor size $>10$ $\mathrm{cm}$. These morphologic criteria apply only partially to rare variants like epitheloid or myxoid LMS. Some cases of dedifferentiated uLMS have been described in analogy to dedifferentiated liposarcoma [20]. These tumors exhibit a smooth-muscle low-grade component and undifferentiated areas without any myogenic cells.

Distinguishing uLMS from leiomyoma (uLM) is crucial with regard to cell-rich uLM, mitotic active uLM, atypical uLM, myxoid
682
Juhasz-Böss/Gabriel/Bohle/Horn/Solomayer/ Breitbach 
uLM, and epitheloid uLM. Precise distinction of these uLM variants from uLMS is impossible in almost $1 / 3$ of the cases [17]. After re-evaluation of $356 \mathrm{uLMS}$ specimens $27 \%$ had to be classified as uLM-variants.

STUMP should be diagnosed only if the histological findings do not match uLMS or uLM or its mentioned variants; so STUMP is a diagnosis of exclusion; essentially only one of the uLMS criteria has to be found, be it cell atypia, mitotic index, or tumor necrosis.

In contrast to uLMS the clinical outcome of most STUMP tumors will be favorable and clinical control after complete resection is sufficient. Immunohistochemical staining does not contribute to differentiation of leiomyogenic tumors, as uLMS as well as uLM will express desmin, h-caldesmon, sm-actin and histone deacetylase (HDCA8). uLMS is often positive for CD10 and epithelial markers like keratin and EMA. Positive reactions are found in 30$40 \%$ with estrogen receptor, progesterone receptor, and androgen receptor; CD117 may be positive, but c-KIT-mutations are not proven [21]. In contrast to uLM a certain amount of uLMS show p53 mutations and overexpression [22].

\section{Adjuvant Systemic Therapy and Radiation}

In general, adjuvant systemic therapy is not indicated outside of clinical studies. At least there are some results of prospective phase II trials and 1 phase III chemotherapy study, which show improved outcome of low-differentiated uLMS confined to the uterus (stage I - IIIa) after complete resection. Compared to historical findings without chemotherapy or with e.g. doxorubicin monotherapy, 3 -year progression-free survival (PFS) of $57 \%$ seems improved by combination chemotherapy with docetaxel / gemcitabine followed by doxorubicin $[23,24]$. Further, a combination of doxorubicin / ifosfamide / cisplatin followed by radiotherapy prompted a reasonable effect on 3-year PFS of 55\% if compared to a control group with radiotherapy alone (41\%). However, it was associated with remarkably higher toxicity [25]. A phase III study (GOG 277) comparing follow-up vs. docetaxel / gemcitabine followed by doxorubicin [24] has been closed due to insufficient accrual and is now awaiting evaluation [26]. Finally, data for adjuvant endocrine therapy of uLMS are lacking.

Adjuvant pelvic radiation with 50.4 Gy provided better local control in a randomized setting of stage I and II uterine sarcoma [27]; but the uLMS subgroup $(\mathrm{n}=99)$ did not profit with regard to local relapse rate (20\% with and $24 \%$ without radiation) nor when overall survival is concerned. In summary radiation therapy of uLMS is not indicated after complete resection of stage I or II tumors. If complete resection cannot be attained $(\mathrm{R} 1, \mathrm{R} 2)$ radiation may be considered in locally advanced tumors confined to the pelvis.

Up to now there are no data reflecting the optimal management of uLMS after accidental morcellation of the tumor.

\section{Therapy of Relapse and/or Metastasis}

Surgery aiming at complete resection seems to be the best strategy when local relapse or metastasis of uLMS is diagnosed. It is associated with better cure rate and prolonged overall survival [2831 ]. In case of local relapse or metastasis the surgical option has to be evaluated regarding a possible complete resection. Some particular studies with selected patient populations demonstrate improved OAS (45 vs. 31 months) after complete resection of uLMS metastasis [31, 32]. Especially resection of single lung or liver metastases may contribute to a prolonged survival $[33,34]$.

Considering palliative single chemotherapy there are only few effective substances, e.g. ifosfamide, gemcitabine, or doxorubicin with moderate remission rates of $15-25 \%[35,36]$. Paclitaxel, cisplatin, topotecan, and etoposide were of insufficient efficacy $(<10 \%)$ [3740]. Only 1 prospective randomized trial shows advantage of a docetaxel / gemcitabine combination versus gemcitabine alone [41], causing considerably higher toxicity. Trabectedin has been investigated as second-line chemotherapy in patients with less symptomatic metastasis. Whereas remission rates have been modest, a nochange situation could be reached for more than 10 cycles in $50 \%$ of patients [42]. Promising results from a French Sarcoma Group trial combining trabectedin with doxorubicin and a randomized trial comparing trabectedin to dacarbazine in soft tissue sarcoma led to FDA approval of trabectedin [3] in advanced and recurrent uLMS.

Immunotherapeutic approaches in soft tissue sarcoma have been reported mainly in first- or second-line palliative setting, including considerable numbers of uLMS patients. Single experiences with PD-1/PDL-1-blockade did not show reasonable effects; addition of bevacizumab did not improve the effect of conventional chemotherapy.

The multi tyrosinkinase inhibitor pazopanib may induce disease stabilization in metastatic uLMS similar to trabectedin and thereby prolong the progression-free interval. A double-blind, placebo-controlled phase III study (PALETTE) included different uterine sarcomas, including 50\% uLMS, and pazopanib significantly prolonged PFS in all patients as well as in the ULMS subgroup [43]. This study led to the FDA approval of pazopanib for uLMS [3].

The PDGFR antibody olaratumab has been studied in a phase II setting combined with doxorubicin versus doxorubicin alone, showing significant prolonged DFS and OAS (11+ months) for the olaratumab combination [44]. The results of this study prompted an accelerated FDA approval in soft tissue sarcoma. Currently a phase III trial (NCT02451943) is ongoing [45].

There are no valid data for the use of endocrine therapies (letrozole, anastrozole) in metastatic LMS, although in individual cases response of metastasis has been reported if estrogen receptors were detected in the tumor tissue.

Percutaneous radiation may be utilized specifically for palliative purposes in cases of unresectable loco-regional relapse [46]. After resection of an isolated single metastasis postoperative radiation may be considered for better local control, but there will be no benefit for overall survival. 


\section{Analysis of Case Report and Discussion}

Do We Have Clinical and Diagnostic Tools to Distinguish Uterine Leiomyoma, STUMP and ULMS?

ULMS mimicks uterine fibroids (uLM), and clinical symptoms and ultrasound findings will not lead to a sufficient distinction between benign and malignant tumors and therefore to a reliable pretherapeutic diagnosis. As the diagnosis of uLMS is rare, additional diagnostic techniques such as MRI, PET-CT, and D\&C or EMB are not commonly used in clinical routine. The number of patients at risk for uLMS has to be narrowed by anamnestic and ultrasound imaging data. Post- or premenopausal patients jounger than 45 years with abnormal uterine bleeding, a history of fast growing uterus and tumor diameter of $>8 \mathrm{~cm}$ should be examined by $\mathrm{D} \& \mathrm{C}$ or EMB, possibly supplemented by more sophisticated imaging techniques. Even with negative biopsy an open complete removal of the intact uterus should be discussed with these patients, if the diameter of the uterus does not allow an endoscopic operation without downsizing by enucleation and power morcellation. Patients not matching this risk group - as in our case report - are in urgent need of an adequate risk score, which has been established but has not been published yet. In addition, a risk score which has been engineered retrospectively needs further prospective confirmation.

If the Diagnosis of uLMS / STUMP Is Made Accidentally, Do We Have Means to Differentiate Accurately between Them?

Given a set of typical histopathologic findings, such as cell-rich spindle-cell areas with cell atypia, high mitotic index, and tumor necrosis the sum of these findings will lead to a diagnosis of uLMS. But in almost one third of cases the diagnosis is ambiguous and has to be downstaged to uLM variants like cell-rich, mitotic active, atypical, myxoid, and epitheloid subtypes. And STUMP should only be diagnosed if there is no sufficient proof for ULM or uLMS. So some of the LMS diagnoses will be overestimated and some of the STUMP underestimated, and the call for a second expert opinion is mandatory. In our case report STUMP diagnosis had been confirmed by second opinion.

\section{How Much of the Tumor Should Be Examined to Minimize} False Negative Histopathological Diagnosis?

As uterine tumors may exhibit large areas with varying histopathologic features, examination of the whole intact tumor is most important. Especially after power morcellation relevant areas of the tumor may not be submitted to the pathologist and thereby hamper a thorough and reliable pathologic diagnosis.

\section{Should the Uterus Be Completely Removed if uLMS or STUMP}

Was Diagnosed? And which Advice Should Be Given if Further Reproduction Is Desired?

An accidental diagnosis of uLMS after a tumor-damaging endoscopic operation should lead to a complete removal of the uterus with careful re-exploration of the abdomial cavity looking for lost residuals from the morcellation process. As uLMS implies the worst prognosis of all uterine tumors, a conservation of the uterus for reproductive reasons means taking more than a high risk. The patient should be strictly advised against planned reproduction.

STUMP shows a considerably better prognosis than uLMS if the uterus has been removed completely. The patient should be advised to undergo a rapid additional second-look operation. There are no reliable data regarding either conversion of STUMP to uLMS or conservation of the enucleated uterus and future pregnancy. If the patient is asking about later reproduction she should be carefully informed of the insufficient data for risk estimation. The process and the decision of the patient should be documented and signed.

In the above case report the patient was diagnosed with STUMP, and after thorough discussion of the ambiguous future outcome she decided to keep the uterus and try to become pregnant. After an interval of less than 1 year she was still on contraception and an uLMS had developed. Although one might speculate that the first diagnosis of STUMP has been incorrect due to missing tumor parts after morcellation, a conversion from STUMP to uLMS seems possible and needs more investigation to broaden the data base for better patient advice.

Under the assumption that morcellation of a uLMS tumor will worsen the prognosis, the FDA released a 'black box' warning in 2014 regarding the use of power morcellators in endoscopic myomectomy or hysterectomy. In the USA this led to a considerable reduction of endoscopic surgery for uterine fibroids during the following years. As the incidence of uLMS is low and many women have profited from endoscopic surgery for myomectomy or hysterectomy, this fall-back to higher rates of open abdominal surgery has been regretted among endoscopic surgeons, and has caused a controversial debate concerning the FDA warning [3]. There is no doubt that risk groups have to be defined more precisely and that there is urgent need for a well evaluated risk score. But even using these tools, accidental morcellation of early uLMS tumors (stage Ia with a diameter of less than $5 \mathrm{~cm}$ confined to the uterus) may not be avoided, and stage Ia is the best prognostic group in uLMS. Therefore attention should be payed to risk analysis of endoscopic in-bag procedures [47] in order to improve safety by means of appropriate tools and operation procedure pathways for patients outside a score-defined risk population.

In the adjuvant situation data for chemo- or endocrine therapy are either disappointing or lacking at all, radiotherapy has been of no benefit for the ULMS subgroup. In case of morcellation or incomplete removal of the tumor the benefit of chemotherapy and / or radiation is not proven and remains a matter of individual consideration. But as long as reports of any adjuvant therapy stay anecdotal there will be no growing knowledge about recommendable strategies.

Therapy studies usually include uterine sarcoma or soft tissue sarcoma although it may not be assumed that all the different types of tumors encountered will behave as a homogeneous group. Obviously this is not true. So even if it will be difficult to manage - like in other rare tumor entities - a widely consented concept of treatment should be established, randomly compared to the recom- 
mended 'wait and see' strategy and offered to any patient diagnosed with uLMS.

Surgery is first-line therapy in recurrent and metastatic disease, if $\mathrm{R} 0$ resection seems to be achievable. Data for systemic palliative treatment of advanced and recurrent tumors offer at least some possibilities, among them chemotherapy with docetaxel and gemcitabine. In addition, some targeted drugs like trabectedin, pazopanib, and olaratumab show promising results. Radiotherapy remains confined to focusses of unresectable tumor residuals.

\section{Conclusion}

The diagnosis of uLMS means poor outcome for most of the patients affected, and complete hysterectomy without tumor laceration and with clean tumor margins is the definite goal of surgical therapy [48]. To decrease the number of incidentally diagnosed uLMS after operations, risk groups have to be defined to prevent detrimental surgical procedures using tumor damage and morcellation. No satisfying and reliable data on a conclusive adjuvant strategy exists, be it chemo-, endocrine or radiotherapy. Therapy recommendations are possible only on an individual basis, affording meticulous decision-sharing with the patient. Palliative treatment is based on restricted chemotherapy data with docetaxel and gemcitabine; up to now outcome is poor. Targeted therapies with trabectedin, pazopanib, or olaratumab offer future promise. All studies suffer by the inclusion of uterine sarcoma or soft tissue sarcoma, which does not allow reliable recommendations for uLMS patients. ULMS should be regarded as an entity of its own. There is a strong need for regional uLMS registration centers, which may offer consented and consistent therapy recommendations to gain more data and knowledge of this rare but threatening disease.

\section{Disclosure Statement}

All authors declare no conflicts of interests.

\section{References}

1 D’Angelo E, Prat J: Uterine sarcomas: a review. Gynecol Oncol 2010;116:131-139.

2 Zaloudek CJ, Hendrickson MR, Soslow RA: Mesenchymal tumors of the uterus; in Kurman RJ, Hedrick Ellenson L, Ronnett BM (eds). Blaustein's Pathology of the Female Genital Tract. New York, Springer, 2011.

3 Ricci S, Stone RL, Fader AN: Uterine leiomyosarcoma: epidemiology, contemporary treatment strategies and the impact of uterine morcellation. Gynecol Oncol 2017;145:208-216.

4 Köhler G, Belau A, Krichbaum J, et al.: Deutsches klinisches Kompetenzzentrum für genitale Sarkome und Mischtumoren an der Universitätsmedizin Greifswald (DKMS) und Kooperationspartner VAAO Deutschland und FK Frankfurt/Sachsenhausen. Datenbank und Promotionsgruppe genitale Sarkome Greifswald 2015. http:// http://www2.medizin.uni-greifswald.de/gyn/forschung/dksm/statistische-daten-des$d k \mathrm{sm} /$ (accessed 2018/10/05).

5 Bansal N, Herzog TJ, Burke W, et al.:The utility of preoperative endometrial sampling for the detection of uterine sarcomas. Gynecol Oncol 2008;110:43.

6 Goto A, Takeuchi S, Sugimura K, et al.: Usefulness of Gd-DTPA contrast-enhanced dynamic MRI and serum determination of $\mathrm{LDH}$ and its isozymes in the differential diagnosis of leiomyosarcoma from degenerated leiomyoma of the uterus, Int. J Gynecol Cancer 2002; 12:354.

7 Sato K, Yuasa N, Fujita M, et al.: Clinical application of diffusion-weighted imaging for preoperative differentiation between uterine leiomyoma and leiomyosarcoma, Am J Obstet Gynecol 2014;210:368.e1-368.e8.

$\checkmark$ Lin G, Yang LY, Huang YT, et al.: Comparison of the diagnostic accuracy of contrast-enhanced MRI and diffusion-weighted MRI in the differentiation between uterine leiomyosarcoma/smooth muscle tumor with uncertain malignant potential and benign leiomyoma. J Magn Reson Imaging 2016;43:333-334.

9 Umesaki N, Tanaka T, Miyama M, et al.: Positron emission tomography with (18)F-fluorodeoxyglucose of uterine sarcoma: a comparison with magnetic resonance imaging and power Doppler imaging. Gynecol Oncol 2001;80:372-377.
10 Köhler G, Evert M, Evert K, et al.: Smooth Muscle and Stromal Tumors. Sarcoma of the Female Genitalia. Vol 1. Berlin, de Gruyter, 2016.

11 Kapp DS, Shin JY, Chan JK: Prognostic factors and survival in 1396 patients with uterine leiomyosarcomas: emphasis on impact of lymphadenectomy and oophorectomy. Cancer 2008;112:820-830.

12 Garg G, Shah JP, Kumar S, et al.: Ovarian and uterine carcinosarcomas: a comparative analysis of prognostic variables and survival outcomes. Int J Gynecol Cancer 2010;20:888-894.

13 Perri T, Korach J, Sadetzki S, et al.: Uterine leiomyosarcoma: does the primary surgical procedure matter? Int J Gynecol Cancer 2009; 19:257-260.

14 Park JY, Park SK, Kim DY, et al.: The impact of tumor morcellation during surgery on the prognosis of patients with apparently early uterine leiomyosarcoma. Gynecol Oncol 2011;122:255-259.

15 Leitao MM, Sonoda Y, Brennan MF, et al.: Incidence of lymph node and ovarian metastases in leiomyosarcoma of the uterus. Gynecol Oncol, 2003;91:209-212.

16 Oduyebo T, Rauh-Hain AJ, et al.: The value of re-exploration in patients with inadvertently morcellated uterine sarcoma. Gynecol Oncol $2014 ; 132: 360-365$.

17 Abeler VM, Røyne O, Thoresen S, et al.: Uterine sarcomas in Norway. A histopathological and prognostic survey of a total population from 1970 to 2000 including 419 patients. Histopathology 2009;54:355-364.

18 Pelmus M, Penault-Llorca F, Guillou L, et al.: Prognostic factors in early-stage leiomyosarcoma of the uterus. Int J Gynecol Cancer 2009;19:385-390.

19 Bell SW, Kempson RL, Hendrickson MR: Problematic uterine smooth muscle neoplasms. A clinicopathologic study of 213 cases. Am J Surg Pathol 1994;18:535-558.

20 Chen E, O'Connell F, Fletcher CD: Dedifferentiated leiomyosarcoma: clinicopathological analysis of 18 cases. Histopathology 2011;59:1135-1143.

21 Raspollini MR, Pinzani P, Simi L, et al.: Uterine leiomyosarcomas express KIT protein but lack mutation(s) in exon 9 of c-KIT. Gynecol Oncol 2005;98:334-335.
2 O’Neill CJ, McBride HA, Connolly LE, et al.: Uterine leiomyosarcomas are characterized by high p16, p53 and MIB1 expression in comparison with usual leiomyomas, leiomyoma variants and smooth muscle tumours of uncertain malignant potential. Histopathology 2007;50:851-858

23 Hensley ML, Maki R, Venkatraman E, et al.: Gemcitabine and docetaxel in patients with unresectable leiomyosarcoma: results of a phase II trial. J Clin Oncol 2002;20:2824-2831.

24 Hensley ML, Wathen JK, Maki RG, et al.: Adjuvant therapy for high-grade, uterus-limited leiomyosarcoma: results of a phase 2 trial (SARC 005). Cancer 2013;119:1555-1561.

25 Pautier P, Floquet A, Gladieff L, et al.: A randomized clinical trial of adjuvant chemotherapy with doxorubicin, ifosfamide, and cisplatin followed by radiotherapy versus radiotherapy alone in patients with localized uterine sarcomas (SARCGYN study). A study of the French Sarcoma Group. Ann Oncol 2013;24:10991104

26 Desar IME, Ottevanger PB, et al.: Systemic treatment in adult uterine sarcoma. Crit Rev Oncol Hematol 2018;122:10-20.

27 Reed NS, Mangioni C, Malmstrom H, et al.: Phase III randomised study to evaluate the role of adjuvant pelvic radiotherapy in the treatment of uterine sarcomas stages I and II: an European Organisation for Research and Treatment of Cancer Gynaecological Cancer Group Study (protocol 55874). Eur J Cancer 2008;44: 808-818.

28 Levenback C, Rubin SC, McCormack PM, et al.: Resection of pulmonary metastases from uterine sarcomas. Gynecol Oncol 1992;45:202-205.

29 Weiser MR, Downey RJ, Leung DH, et al.: Repeat resection of pulmonary metastases in patients with softtissue sarcoma. J Am Coll Surg 2000;191:184-190; discussion 190-181.

30 Leitao MM, Brennan MF, Hensley M, et al.: Surgical resection of pulmonary and extrapulmonary recurrences of uterine leiomyosarcoma. Gynecol Oncol 2002;87:287-294. 
31 Bernstein-Molho R, Grisaro D, Soyfer V, et al.: Metastatic uterine leiomyosarcomas: a single-institution experience. Int J Gynecol Cancer 2010;20:255-260.

32 Giuntoli RL, 2nd, Garrett-Mayer E, Bristow RE, et al.: Secondary cytoreduction in the management of recurrent uterine leiomyosarcoma. Gynecol Oncol 2007; 106:82-88.

33 Chen H, Pruitt A, Nicol TL, et al.: Complete hepatic resection of metastases from leiomyosarcoma prolongs survival. J Gastrointest Surg 1998;2:151-155.

34 Anraku M, Yokoi K, Nakagawa K, et al.: Pulmonary metastases from uterine malignancies: results of surgical resection in 133 patients. J Thorac Cardiovasc Surg 2004;127:1107-1112.

35 Sutton GP, Blessing JA, Barrett RJ, et al.: Phase II trial of ifosfamide and mesna in leiomyosarcoma of the uterus: a Gynecologic Oncology Group study. Am J Obstet Gynecol 1992;166:556-559.

36 Look KY, Sandler A, Blessing JA, et al.: Phase II trial of gemcitabine as second-line chemotherapy of uterine leiomyosarcoma: a Gynecologic Oncology Group (GOG) Study. Gynecol Oncol 2004;92:644-647.

37 Thigpen T, Blessing JA, Yordan E, et al.: Phase II trial of etoposide in leiomyosarcoma of the uterus: a Gynecologic Oncology Group study. Gynecol Oncol 1996;63:120-122.
38 Rose PG, Blessing JA, Soper JT, et al.: Prolonged oral etoposide in recurrent or advanced leiomyosarcoma of the uterus: a gynecologic oncology group study. Gynecol Oncol 1998;70:267-271.

39 Miller DS, Blessing JA, Kilgore LC, et al.: Phase II trial of topotecan in patients with advanced, persistent, or recurrent uterine leiomyosarcomas: a Gynecologic Oncology Group Study. Am J Clin Oncol 2000;23:355357.

40 Gallup DG, Blessing JA, Andersen W, et al.: Evaluation of paclitaxel in previously treated leiomyosarcoma of the uterus: a gynecologic oncology group study. Gynecol Oncol 2003;89:48-51

41 Maki RG, Wathen JK, Patel SR, et al.: Randomized phase II study of gemcitabine and docetaxel compared with gemcitabine alone in patients with metastatic soft tissue sarcomas: results of sarcoma alliance for research through collaboration study 002 [corrected]. J Clin Oncol 2007;25:2755-2763.
42 Monk BJ, Blessing JA, Street DG, et al.: A phase II evaluation of trabectedin in the treatment of advanced, persistent or recurrent uterine leiomyosarcoma: a Gynecologic Oncology Group study. Gynecol Oncol 2012;124:48-52.

43 van der Graaf WT, Blay JY, Chawla SP, et al.: Pazopanib for metastatic soft-tissue sarcoma (PALETTE): randomised, double-blind, placebo-controlled phase 3 trial. Lancet 2012;379:1879-1886.

44 Tap WD, Jones RL, Van Tine BA, et al.: Olaratumab and doxorubicin versus doxorubicin alone for treatment of softtissue sarcoma: an open-label phase $1 \mathrm{~b}$ and randomised phase 2 trial. Lancet 2016;388:488-497.

45 Tobias A, O'brien MP, Agulnik M: Olaratumab for advanced soft tissue sarcoma. Expert Rev Clin Pharmacol 2017;10:699-705.

46 Dusenbery KE, Potish RA, Judson P: Limitations of adjuvant radiotherapy for uterine sarcomas spread beyond the uterus. Gynecol Oncol 2004;94:191-196.

47 van den Haak L, van der Eijk AC, Sandberg EM, et al.: Towards spill-free in-bag morcellation: a health failure mode and effects analysis. Surg Endosc 2018doi: 10.1007/s00464-018-6284-z. [Epub ahead of print].

48 Sarcoma of the uterus. Guideline of German Society of Gynecology and Obstetrics (S2k-Level, AWMF Registry No. 015/074, August 2015). http://www.awmf.org/ leitlinien/detail/ll/015074.html 